\title{
Quantum Mechanics and Special Relativity: The Origin of Momentum Operator
}

\author{
Gintautas P. Kamuntavičius \\ Vytautas Magnus University, Kaunas, Lithuania \\ Email: gintautas.kamuntavicius@vdu.lt
}

How to cite this paper: Kamuntavičius, G.P. (2018) Quantum Mechanics and Special Relativity: The Origin of Momentum Operator. Journal of Applied Mathematics and Physics, 6, 2006-2023.

https://doi.org/10.4236/jamp.2018.610172

Received: September 25, 2018

Accepted: October 21, 2018

Published: October 24, 2018

Copyright $\odot 2018$ by author and Scientific Research Publishing Inc. This work is licensed under the Creative Commons Attribution International License (CC BY 4.0).

http://creativecommons.org/licenses/by/4.0/

\begin{abstract}
The free relativistic particle, by definition, has to move in an inertial reference frame with uniform velocity less than the speed of light. The corresponding movement of a material quantum particle describes a wave packet, composed of matter waves-solutions of the Schrödinger equation. The maximum of packet, corresponding to the largest probability to find the particle, has to move with the same uniform velocity, defined by the initial condition. It has been shown that the traditional definition of the quantum momentum operator i.e. taking it to correspond to the special relativity theory, relativistic momentum, cannot produce precise description of a relativistic matter particle. Different definitions are investigated and one that solves this issue is found. Obtained original expression of relativistic kinetic energy operator creates new possibilities for relativistic quantum systems theory.
\end{abstract}

\section{Keywords}

Special Relativity, Quantum Mechanics, Relativistic Wave Equations

\section{Introduction}

The fundamental and at the first glance the simplest problem of quantum mechanics is description of moving in vacuum free particle. However, careful investigation of this problem has shown that well known and present in numerous textbooks, recipes for this task solution are not successful and have to be corrected.

Traditionally, the quantum mechanical momentum operator $-i \hbar \nabla$ is associated with the classical relativistic momentum $p=\gamma m v$, present in the energy expression

$$
E=\sqrt{m^{2} c^{4}+p^{2} c^{2}}
$$


where, as usual, $\gamma=1 / \sqrt{1-v^{2} / c^{2}}$. The main argument for this association is the photon energy definition $E=p c$, producing after Schrödinger equation solution the electromagnetic waves, whose packet moves with the speed of light and defines the probability distribution of a photon.

However, this definition, applied to a free particle, whose $m \neq 0$, leads to a result not consistent with fundamental conditions, following from the inertial reference system definition, stating that a free particle in an arbitrary, such system has to move with uniform velocity, defined by the initial condition and less than speed of light. The maximum of the packet, composed of matter waves-solutions of Schrödinger equation-has to move specifically with this velocity. Moreover, the traditional definition of the momentum operator produces a kinetic energy operator, present in the Schrödinger equation, whose expectation values at any momentum give upper bounds for relativistic kinetic energy, larger than the corresponding nonrelativistic energy and not consistent with this equation's definition as nonrelativistic.

Another definition of this operator as corresponding to the nonrelativistic classical momentum $p_{0}=m v$ restores nonrelativity of Schrödinger equation, because the corresponding kinetic energy operator expectation values are equal, as necessary, to the lower bounds of relativistic kinetic energy. However, the expectation values of this operator have to satisfy the condition for classical nonrelativistic momentum to be less than $m c$. As a consequence, the nonrelativistic matter wave packet, satisfying mentioned conditions, cannot be obtained integrating matter waves in the finite region $\left(-m c<p_{0}<m c\right)$ of momentum.

The objective of this work is to examine the consequences of different definitions of the quantum momentum operator's correspondence to classical parameters with momentum dimension and to present a developed problem solving approach.

In Section II, we provide the problem, applied terms and notations. In Section III, the matter waves and packets produced during the application of the quantum momentum operator, corresponding to the classical momentum, are investigated. Section IV is devoted to the traditional model with this operator corresponding to the relativistic classical momentum. From our considerations it follows that both definitions cannot produce packets, satisfying the mentioned conditions. A problem-solving method is presented in Section V. The characteristic feature of the method is based on the defined original correspondence of quantum mechanical momentum operator to a new parameter of classical relativity theory, producing a simple expression of relativistic kinetic energy operator. The conclusions provide the recommendations for the developed method application.

\section{Formulation of the Problem}

Really, the only particles, not having yet the known structure, are quarks, the photon and leptons. Protons, neutrons, atomic nuclei, ions of atoms and 
molecules, moving in colliders and cosmos, are bound quantum systems, whose center of inertia (center of mass) moves as particle. Therefore, the relativistic generalization of Schrödinger equation has to start with one written in a laboratory reference frame for a many-particle quantum system

$$
i \hbar \frac{\partial}{\partial t} \Psi\left(\rho_{1}, \rho_{2}, \cdots, \rho_{N}, t\right)=\hat{H}_{0}\left(\rho_{1}, \rho_{2}, \cdots, \rho_{N}\right) \Psi\left(\rho_{1}, \rho_{2}, \cdots, \rho_{N}, t\right) .
$$

Here $\rho_{j}$ marks the set of one-particle variables (spatial radius vector $\boldsymbol{r}_{j}$ and internal degrees of freedom $\tau_{j}$ like mass, charge, spin, isospin and so on for every particle). As usual, the operators are marked with accent above the letter and vectors are present in bold face. The Hamiltonian is independent of time, so the formal solution of (2) is

$$
\Psi\left(\rho_{1}, \rho_{2}, \cdots, \rho_{N}, t\right)=\exp \left(-\frac{i}{\hbar} \hat{H}_{0} t\right) \Phi\left(\rho_{1}, \rho_{2}, \cdots, \rho_{N}\right) .
$$

The stationary states of (2) correspond to the eigenfunctions of the Hamiltonian:

$$
\hat{H}_{0} \Phi=E \Phi .
$$

The stationary Schrödinger Equation (4) is written in a laboratory reference frame. Even in the nonrelativistic approximation the wave functions dependent on one-particle spatial variables are not good enough for a precise description of the quantum system. These do not satisfy Galilean invariance and, in many cases, the wave functions of this model contain uncontrolled center of mass excitations. For the best illustration of this statement, let us consider a two-particle system. The potential energy operator is translationally invariant, i.e. independent of the center of mass coordinate, hence the only problem is the kinetic energy. No one applies the corresponding wave function written in one-particle spatial variables, because the elimination of the center of mass kinetic energy operator in a two-particle Hamiltonian is an easy task. Elimination of the center of mass kinetic energy operator is not complicated for a system of $N$ particles either. However, after this the Hamiltonian of the quantum system appears as a function of $3(N-1)$ internal spatial variables and $N$ sets of individual degrees of freedom of composing the system (or some subsystem like electrons in atom) identical fermions, hence the anti-symmetrization of eigenfunctions of this operator becomes a very complex problem (see [1] and [2]). However, after long investigations the simplest possible solution of this problem, reducing significantly the dimensions of basic functions, present in expansions, was found [3]. Thus, the principal problems cannot appear, and for the center of mass of quantum system separation one needs to perform the transformation of operator present in the stationary Schrödinger equation to internal Hamiltonian.

For a quantum system composed of point particles with pair interaction, the Hamiltonian is

$$
H_{0}\left(\boldsymbol{r}_{1} \tau_{1}, \boldsymbol{r}_{2} \tau_{2}, \cdots, \boldsymbol{r}_{N} \tau_{N}\right)=-\sum_{j=1}^{N} \frac{\hbar^{2}}{2 m_{j}} \Delta_{j}+\sum_{k>j=1}^{N} V\left(\boldsymbol{r}_{k}-\boldsymbol{r}_{j}, \tau_{j} \tau_{k}\right) .
$$


As usual, for every particle here is present only the first member of relativistic kinetic energy $T=E-m c^{2}$ expansion

$$
\begin{aligned}
T & =\gamma m c^{2}-m c^{2}=m c^{2}\left[\left(1-\left(p_{0} / m c\right)^{2}\right)^{-1 / 2}-1\right] \\
& =m c^{2} \sum_{k=1}^{\infty}\left(\begin{array}{c}
2 k \\
k
\end{array}\right)\left(\frac{p_{0}}{2 m c}\right)^{2 k}=\frac{p_{0}^{2}}{2 m}+\frac{3 p_{0}^{4}}{8 m^{3} c^{2}}+\cdots
\end{aligned}
$$

applying instead of classical momentum the quantum mechanical momentum operator

$$
\hat{p}_{0}=-i \hbar \nabla .
$$

The classical momentum is defined in the usual way $p_{0}=m v$, where $v$ is the velocity of the particle in the laboratory reference frame.

Having in mind that one has to generalize the Schrödinger equation for relativistic dynamics, it is useful to add the rest energies of particles to the Hamiltonian. The modified Hamiltonian is

$$
\hat{H}\left(\boldsymbol{r}_{1} \tau_{1}, \boldsymbol{r}_{2} \tau_{2}, \cdots, \boldsymbol{r}_{N} \tau_{N}\right)=\sum_{j=1}^{N}\left(m_{j} c^{2}-\frac{\hbar^{2}}{2 m_{j}} \Delta_{j}\right)+\sum_{k>j=1}^{N} \hat{V}\left(\boldsymbol{r}_{k}-\boldsymbol{r}_{j}, \tau_{j} \tau_{k}\right) .
$$

Now one can separate the center of mass and internal Hamiltonians. The best choice are internal Jacobi variables, defined as vectors connecting the centers-of-mass of two clusters of particles, while the reduced mass, corresponding to the given coordinate is defined by the conventional expression, using masses of clusters. The Jacobi variable system is generated by splitting down the system and later on each subsystem of single particle coordinates into two clusters with any number of particles. For example, if the system of $N$ particles is split into clusters, where first contains the first $k$ and second-the rest $N-k$ coordinates, corresponding Jacobi variable is defined as

$$
\boldsymbol{\xi}_{\alpha}=\frac{1}{M_{1}} \sum_{j=1}^{k} m_{j} \boldsymbol{r}_{j}-\frac{1}{M_{2}} \sum_{j=k+1}^{N} m_{j} \boldsymbol{r}_{j},
$$

where

$$
M_{1}=\sum_{j=1}^{k} m_{j}, \quad M_{2}=\sum_{j=k+1}^{N} m_{j},
$$

and, as mentioned above, corresponding reduced mass equals

$$
\mu_{\alpha}=\frac{M_{1} M_{2}}{M_{1}+M_{2}} .
$$

Defined in this way Jacobi variables $\xi_{\alpha}(\alpha=1, \cdots, N-1)$ together with center of mass radius vector

$$
\boldsymbol{\xi}_{0}=\frac{1}{\mu_{0}} \sum_{j=1}^{N} m_{j} \boldsymbol{r}_{j}
$$

and sum of particles masses as corresponding reduced mass

$$
\mu_{0}=\sum_{j=1}^{N} m_{j}
$$


compose the system of variables, transforming the kinetic energy operator, present in $\hat{H}$, to analogous expression without mixed derivatives:

$$
\sum_{j=1}^{N} \frac{1}{m_{j}} \Delta_{j}=\sum_{\alpha=0}^{N-1} \frac{1}{\mu_{\alpha}} \Delta_{(\alpha)} .
$$

Now the $\hat{H}$ takes the form with separated variables

$$
\hat{H}=-\frac{\hbar^{2}}{2 \mu_{0}} \Delta_{(0)}+\hat{H}_{i n t},
$$

where the internal Hamiltonian is defined as

$$
\hat{H}_{\text {int }}=-\sum_{\alpha=1}^{N-1} \frac{\hbar^{2}}{2 \mu_{\alpha}} \Delta_{(\alpha)}+\mu_{0} c^{2}+\hat{W}\left(\xi_{1} \xi_{2} \cdots \xi_{N-1} \tau_{1} \tau_{2} \cdots \tau_{N}\right) .
$$

$\hat{W}$ is potential energy operator, dependent only on intrinsic spatial variables.

Having in mind that eigenvalues of $\hat{H}_{i n t}$ without rest mass $\mu_{0} c^{2}$ equal the binding energy $B$ with opposite sign and the quantum system is stable with respect to breakup into composing particles if $B$ is positive, the eigenvalue of modified Hamiltonian corresponds to the rest energy of quantum system under consideration $\mu c^{2}=\mu_{0} c^{2}-B$. Due to binding produced by interaction it is less than sum of rest energies of particles $\mu_{0} c^{2}$.

Later on we will consider the application of relativistic dynamics to quantum systems defined this way. All particles, composing the system, move in the same inertial frame, hence any transformations of radius vector and time coordinate or energy and momentum of a given particle between different inertial systems are not necessary. The energy of a stationary state is defined precisely, hence the relativistic energy and momentum conservation law is senseless in a fixed reference frame defined for a quantum system by its internal Hamiltonian. Moreover, at a precisely defined energy the time coordinate is completely uncertain, therefore it is not necessary as an argument of wave function. At the same time in formalisms, operating with one particle coordinates, all inertial systems have to be equivalent and invariance of formalism with respect to Lorentz transformations is necessary. The space-time interval, defined by the scalar product of four-dimensional vectors and invariant with respect to transformations between different inertial frames, requires every particle to have different spatial and time variables. The problems of wave functions presented this way are well known (lattice QCD; for a review, see [4]). In our formalism we don't have to worry about interval and mass invariance in different inertial reference systems. Instead, the only problem is modification of the internal Hamiltonian by introducing the relativistic kinetic energy operator instead of the nonrelativistic one for every internal spatial coordinate.

Thus, after these modifications the Schrödinger equation can be written as

$$
\left(i \hbar \frac{\partial}{\partial t}+\frac{\hbar^{2}}{2 \mu_{0}} \Delta_{(0)}-\hat{H}_{i n t}\right) \Psi\left(\xi_{1} \xi_{2} \cdots \boldsymbol{\xi}_{N-1} \tau_{1} \tau_{2} \cdots \tau_{N}, \boldsymbol{\xi}_{0} t\right)=0 .
$$

Again, this operator is with separated variables, hence the eigenfunctions are 
equal the products of two functions-one dependent on set of internal variables and other-dependent on center of mass radius-vector and time, i.e.

$$
\Psi\left(\xi_{1} \xi_{2} \cdots \xi_{N-1} \tau_{1} \tau_{2} \cdots \tau_{N}, \xi_{0} t\right)=\psi\left(\xi_{1} \xi_{2} \cdots \xi_{N-1} \tau_{1} \tau_{2} \cdots \tau_{N}\right) \phi\left(\xi_{0} t\right) .
$$

Here $\psi$ is eigenfunction of $\hat{H}_{\text {int }}$, corresponding to the eigenvalue, equal to the rest energy of system

$$
\hat{H}_{i n t} \psi=\mu c^{2} \psi
$$

and $\phi$ is an eigenfunction of the operator, dependent on time and center of mass radius-vector, corresponding the same eigenvalue:

$$
\left(i \hbar \frac{\partial}{\partial t}+\frac{\hbar^{2}}{2 \mu_{0}} \Delta_{(0)}\right) \phi=\mu c^{2} \phi \text {. }
$$

The last modification of this equation is the exchange of mass $\mu_{0}$, present in the nonrelativistic kinetic energy operator and equal to the sum of masses of particles, with the mass of quantum system $\mu$, obtained after equation with intrinsic Hamiltonian (19) solution or by real rest mass of quantum system under investigation. Finally, the equation, describing the movement of the center of mass takes the form

$$
i \hbar \frac{\partial}{\partial t} \phi=\left(\mu c^{2}-\frac{\hbar^{2}}{2 \mu} \Delta_{(0)}\right) \phi
$$

The operator, present in right-hand side of this equation, equals the nonrelativistic approximation of the energy of the particle. The generalization of this equation for relativistic dynamics is

$$
i \hbar \frac{\partial}{\partial t} \phi\left(\xi_{0} t\right)=\hat{H}\left(\xi_{0}\right) \phi\left(\xi_{0} t\right) .
$$

This equation looks exactly as the well-known Schrödinger equation for a free particle, however now it is obtained by separating the center of mass and intrinsic parts of quantum system's Hamiltonian. The introduced internal Hamiltonian, whose eigenvalues are equal to the rest energy of the system, is separated from the center of mass degree of freedom, whose movement is independent of intrinsic excitations of quantum system. The developed technique is useful for precise separation of center of mass and internal degrees of freedom when quantum system enters an external field. Moreover, the Equation (22) is basic for further modifications and relativistic dynamics of free particle investigation taking different expressions of the free particle energy operator $\hat{H}\left(\xi_{0}\right)$.

Let me present here the slightly modified, in comparison with traditional consideration (see [5], [6], [7]), main points of this solution, necessary for further modifications, taking into account relativistic dynamics.

The Hamiltonian $\hat{H}\left(\xi_{0}\right)$ is independent of time, hence the fundamental system of Equation (22) solutions are products of eigenfunctions of two operators, present in opposite sides of equation, corresponding to the same eigenvalue: 


$$
\phi\left(\xi_{0} t\right)=\chi_{E}\left(\xi_{0}\right) \eta_{E}(t)
$$

where

$$
\hat{H}\left(\boldsymbol{\xi}_{0}\right) \chi_{E}\left(\boldsymbol{\xi}_{0}\right)=E \chi_{E}\left(\boldsymbol{\xi}_{0}\right)
$$

and

$$
i \hbar \frac{\partial}{\partial t} \eta_{E}(t)=E \eta_{E}(t)
$$

The last modification is presentation of relativistic energy in terms of momentum. Introducing in this expression instead of momentum the quantum mechanical momentum operator creates differential equations for mentioned fundamental solutions. Obviously, the square-integrable and satisfying the initial condition solution of Equation (22), giving the free quantum particle description, can be expressed as a linear combination of these fundamental solutions.

The easiest energy expression in terms of momentum is for a particle whose rest mass equals zero. In special relativity theory it is defined as $E=|\boldsymbol{p}| c$. Therefore the corresponding operator is

$$
\hat{H}\left(\boldsymbol{\xi}_{0}\right)=-i \hbar c \nabla_{(0)}
$$

and the wave function equals

$$
\phi\left(\boldsymbol{\xi}_{0} t\right)=\exp \left[-\frac{i}{\hbar}\left(p c t-\left(\boldsymbol{p} \cdot \boldsymbol{\xi}_{0}\right)\right)\right] .
$$

By definition, the inertial reference frame is defined as one, where the free particle moves with uniform velocity. Taking the $z$ axis of the reference frame to be along the vector $p$, one finds that

$$
\phi_{p}(z t)=\exp \left[-\frac{i}{\hbar} p(c t-z)\right],
$$

where $z=\left(\xi_{0}\right)_{z}$. The phase velocities of these harmonic plane waves are independent of $p$ and equal the speed of light. The linear combination of waves

$$
\psi(z t)=\int_{-\infty}^{+\infty} f(p) \phi_{p}(z t) \mathrm{d} p
$$

represents the wave function of the relativistic particle, whose rest mass equals zero. The maximum of the probability distribution $|\psi(z t)|^{2}$ of the square integrable packet moves with the velocity of the particle, equal to the speed of light.

For a particle, whose rest mass does not equal zero, there are different energy expressions in terms of momentum and possible different momentum definitions, therefore this problem needs separate investigation.

\section{Matter Waves and Packets with Nonrelativistic Momentum Operator}

Let us start the investigation of Equation (21) for a nonrelativistic free particle. The corresponding Hamiltonian equals the nonrelativistic approximation of the energy expression 


$$
E=\frac{\mu c^{2}}{\sqrt{1-\left(\frac{p_{0}}{\mu c}\right)^{2}}}=\mu c^{2}+\frac{p_{0}^{2}}{2 \mu}+\cdots,
$$

where $\boldsymbol{p}_{0}=\mu \boldsymbol{v}$ is the classical momentum of the particle. Defining the quantum mechanical operator $-i \hbar \nabla$ as corresponding to the classical momentum, the nonrelativistic Hamiltonian of a free particle is

$$
\hat{H}\left(\xi_{0}\right)=\mu c^{2}+\frac{\hat{p}_{0}^{2}}{2 \mu} .
$$

Eigenfunctions of this operator coincide with eigenfunctions of the momentum operator $\hat{\boldsymbol{p}}_{0}$ and equal

$$
\exp \left[-\frac{i}{\hbar}\left(\mu c^{2}+\frac{p_{0}^{2}}{2 \mu}\right) t\right]
$$

Here $\boldsymbol{p}_{0}$ is the eigenvalue of the momentum operator. The matter wave is

$$
\phi_{p_{0}}\left(\boldsymbol{\xi}_{0} t\right)=\exp \left(-\frac{i}{\hbar} \mu c^{2} t\right) \exp \left[\frac{i}{\hbar}\left(\left(\boldsymbol{p}_{0} \cdot \boldsymbol{\xi}_{0}\right)-\frac{p_{0}^{2}}{2 \mu} t\right)\right] \text {. }
$$

The wave function of the packet, satisfying the normalization condition

$$
\int\left|\psi\left(\xi_{0} t\right)\right|^{2} \mathrm{~d} \xi_{0}=1
$$

equals a linear combination of matter waves:

$$
\psi\left(\boldsymbol{\xi}_{0} t\right)=\int f\left(\boldsymbol{p}_{0}\right) \phi_{\boldsymbol{p}_{0}}\left(\boldsymbol{\xi}_{0} t\right) \mathrm{d} \boldsymbol{p}_{0} .
$$

Let us mark the components of $\xi_{0}$ as $x, y$ and $z$. The spectral function $f\left(p_{0}\right)$ can be defined by the initial condition

$$
\psi\left(\boldsymbol{\xi}_{0} 0\right)=N \exp \left(-\frac{x^{2}}{4 \sigma_{x}^{2}}-\frac{y^{2}}{4 \sigma_{y}^{2}}-\frac{z^{2}}{4 \sigma_{z}^{2}}\right) \exp \left[\frac{i}{\hbar}\left(\boldsymbol{p}_{0 i} \cdot \boldsymbol{\xi}_{0}\right)\right] .
$$

Here $N$ is the norm, $\sigma_{x}, \sigma_{y}$ and $\sigma_{z}$ are spatial extensions of the packet along axes of the reference frame and $\boldsymbol{p}_{0 i}=\mu \boldsymbol{v}$ is the momentum of the initial packet, defining the center of mass velocity. As mentioned, a free particle in an arbitrary inertial reference frame moves with constant velocity, hence the initial condition can be simplified taking the direction of $z$ axis of the reference system to coincide with the direction of the initial momentum $\boldsymbol{p}_{0 i}$. Now the initial condition converts to the normalized one-dimensional form:

$$
\psi(z 0)=\frac{1}{\sqrt{\sigma \sqrt{2 \pi}}} \exp \left(-\frac{z^{2}}{4 \sigma^{2}}\right) \exp \left[\frac{i}{\hbar}\left(p_{0 i} z\right)\right],
$$

because the momentum projections, hence also the corresponding extensions along $x$ and $y$ axes equal zeroes. According to Heisenberg principle, these spatial extensions approach infinity, hence the corresponding exponents of the initial condition equals unities. For the sake of simplicity here $\sigma_{z}$ is marked without subscript as $\sigma$. 
The spectral function $f\left(\boldsymbol{p}_{0}\right)$ after this suggestion is not correlated along different axes, hence presentable as product of components:

$$
f\left(\boldsymbol{p}_{0}\right)=f_{x}\left(p_{0 x}\right) f_{y}\left(p_{0 y}\right) f\left(p_{0}\right) .
$$

From initial condition for packet wave function it follows:

$$
\left\{\begin{array}{l}
f_{x}\left(p_{0 x}\right)=\delta\left(p_{0 x}\right) \\
f_{y}\left(p_{0 y}\right)=\delta\left(p_{0 y}\right) .
\end{array}\right.
$$

Normalized in necessary way the spectral function equals

$$
f\left(p_{0}\right)=\sqrt{\frac{\sigma \sqrt{2 \pi}}{\pi \hbar}} \exp \left(-\frac{\sigma^{2}\left(p_{0}-p_{0 i}\right)^{2}}{\hbar^{2}}\right) .
$$

From this exponent, present as

$$
\exp \left(-\frac{\left(p_{0}-p_{0 i}\right)^{2}}{4 \sigma_{p}^{2}}\right)
$$

it follows that momentum extension equals

$$
\sigma_{p}=\frac{\hbar}{2 \sigma}
$$

hence the product of spatial and momentum extensions satisfy Heisenberg's uncertainty relation

$$
\sigma \sigma_{p} \geq \frac{\hbar}{2}
$$

The wave function of packet (35) after explicit integration takes the form

$$
\psi(z, t)=\frac{1}{\sqrt{\sigma(t) \sqrt{2 \pi}}} \exp \left(-\frac{(z-v t)^{2}}{4 \sigma^{2}(t)}\right) \exp (i \phi(z, t)) .
$$

The probability density distribution $|\psi(z, t)|^{2}$ is

$$
\frac{1}{\sigma(t) \sqrt{2 \pi}} \exp \left(-\frac{(z-v t)^{2}}{2 \sigma^{2}(t)}\right) \text {. }
$$

The spatial extension of the packet

$$
\sigma(t)=\sigma \sqrt{1+\left(\frac{\hbar t}{2 \mu \sigma^{2}}\right)^{2}}
$$

increases with time. The function $\phi(z, t)$ determining the phase of the carrier wave, equals

$$
\begin{aligned}
\phi(z, t)= & \frac{1}{\hbar}\left(\frac{\mu v^{2}}{2} t-\mu \sigma^{2} t+\left(p_{0 i}+\frac{\hbar^{2} t}{8 \mu \sigma^{2} \sigma^{2}(t)}(z-v t)\right)(z-v t)\right) \\
& -\frac{1}{2} \arctan \frac{\hbar t}{2 \mu \sigma^{2}} .
\end{aligned}
$$

The description of a free nonrelativistic particle, obtained without any approximations after explicit integration of fundamental quantum mechanical 
expressions, gives the best possible result predicting comparable with experimental observations spreading of packet and its maximum, moving with the velocity of particle.

This result, together with arguments about the origin of quantum mechanical momentum operator (independence of speed of light; suggested by Dirac derivation from classical Poisson brackets; de Broglie waves, defined as solutions of Schrödinger equation with momentum operator, corresponding the classical momentum of particle; definition of momentum operator as translations in three-dimensional space generator), present in [8], shows that the quantum momentum operator $-i \hbar \nabla$ corresponds to the classical $\left(\boldsymbol{p}_{0}=m \boldsymbol{v}\right)$ rather than the relativistic $\left(\boldsymbol{p}=\gamma \boldsymbol{p}_{0}\right)$ momentum. Moreover, this correspondence defines the kinetic energy operator, present in the Schrödinger equation, as a completely nonrelativistic operator. The expectation value of the relativistic kinetic energy, as necessary, is larger than the corresponding nonrelativistic expectation value.

However, the deeper investigation and an attempt to apply it for the relativistic free particle has shown that the defined momentum operator correspondence is not so successful. The problem is that (45) produces a wave packet for all values of the particle's nonrelativistic momentum, including velocities larger than the speed of light. At the same time the relativistic energy operator (30) is [8]:

$$
\hat{H}=\mu c^{2} \sum_{k=0}^{\infty}\left(\begin{array}{c}
2 k \\
k
\end{array}\right)\left(\frac{\hat{\boldsymbol{p}}_{0}}{2 \mu c}\right)^{2 k} .
$$

It is present in terms of the momentum operator, hence both have the same system of eigenfunctions

$$
\exp \left(\frac{i}{\hbar}\left(\boldsymbol{p}_{0} \cdot \boldsymbol{\xi}_{0}\right)\right)
$$

with different eigenvalues. As mentioned, if the $\hat{\boldsymbol{p}}_{0}$ eigenvalue is $\boldsymbol{p}_{0}$, the eigenvalue of $\hat{H}$ equals

$$
\mu c^{2} \sum_{k=0}^{\infty}\left(\begin{array}{c}
2 k \\
k
\end{array}\right)\left(\frac{p_{0}}{2 \mu c}\right)^{2 k}=\mu c^{2} / \sqrt{1-\left(\frac{p_{0}}{\mu c}\right)^{2}} .
$$

The problem, following from this expression, is that the momentum eigenvalue $p_{0}$ for a matter particle has to be less than $\mu c$. The consequences of this condition for two particle quantum systems with different potentials are considered in [9]. This condition also changes significantly the obtained nonrelativistic result for the free particle, because the integration over $\boldsymbol{p}_{0}$ is performed using infinite limits of integrals. Taking this into account produces a packet not corresponding to the main condition that the maximum of the packet has to move with a uniform initial velocity of the particle. The velocity of the packet maximum appears as a nonlinear function of parameters $p_{0 i}$ and $\sigma$ defined by the initial condition.

Now let us investigate the relativistic particle description. The relativistic kinetic energy operator, expressed in terms of the nonrelativistic momentum operator, is 


$$
\hat{T}\left(\hat{\boldsymbol{p}}_{0}\right)=\mu c^{2} \sum_{k=1}^{\infty}\left(\begin{array}{c}
2 k \\
k
\end{array}\right)\left(\frac{\hat{\boldsymbol{p}}_{0}}{2 \mu c}\right)^{2 k} .
$$

Now the matter wave, defined like one for the nonrelativistic packet, equals

$$
\phi_{p_{0}}(z t)=\exp \left(-\frac{i}{\hbar} \mu c^{2} t\right) \exp \left[\frac{i}{\hbar} p_{0} z-T\left(p_{0}\right) t\right] .
$$

Definitions of the packet wave function (35) and initial condition (37) are the same as for the nonrelativistic particle, hence the same expressions are obtained for spectral functions (39) and (40). However, now the analytical presentation of the packet wave function is not possible, but simple enough numerical integration gives complete information about the movement of the wave packet.

This investigation has shown that the construction of a packet, moving in a given reference frame with uniform initial velocity, is not possible for this definition of the momentum operator. The velocity of the maximum of the packet appears dependent in a nonlinear way on the initial momentum $p_{0 i}$. Another phenomenon is caused by fact that at small values of $\sigma$, present in the spectral function (40), the tile of the Gausoid enters the forbidden momenta region $p_{0} \geq \mu c$, and the packet's expansion loses corresponding momenta. As a result, the velocity of the packet does not correspond to the particle's velocity. At different combinations of these parameters it can be smaller or larger than necessary.

Concluding, one has to state that the nonrelativistic quantum momentum operator definition is not applicable to the description of a free particle, moving in an inertial reference frame.

\section{Matter Waves and Packets with Relativistic Momentum Operator}

Now we will apply another possibility-relativistic energy presentation in terms of the relativistic momentum like the one applied for a photon. This can be derived from the fundamental energy definition $E=\gamma \mu c^{2}$, after presenting the factor $\gamma$ in terms of the relativistic momentum $p$ instead of the known presentation in terms of $p_{0}$ like in Equation (30). From the definition of the relativistic momentum,

$$
p=\frac{p_{0}}{\sqrt{1-\left(\frac{p_{0}}{\mu c}\right)^{2}}}
$$

it follows

$$
p_{0}=\frac{p}{\sqrt{1+\left(\frac{p}{\mu c}\right)^{2}}} .
$$

Having in mind that by definition $p=\gamma p_{0}$, we get the following expression 
of $\gamma$

$$
\gamma=\sqrt{1+\left(\frac{p}{\mu c}\right)^{2}} .
$$

Therefore, the relativistic energy can be present as

$$
E=\mu c^{2} \sqrt{1+\left(\frac{p}{\mu c}\right)^{2}} .
$$

The square of this expression gives the well known

$$
E^{2}=\mu^{2} c^{4}+p^{2} c^{2}
$$

however we will apply (56) to avoid problems with an undefined sign taking square root of the squared expression.

The converging at $p \leq \mu c$ series expansion of the energy in terms of the relativistic momentum is

$$
E=\mu c^{2}+\frac{p^{2}}{2 \mu}+\frac{p^{2}}{\mu} \sum_{k=1}^{\infty}(-1)^{k} \frac{(2 k-1) ! !}{(2 k+2) ! !}\left(\frac{p}{\mu c}\right)^{2 k} .
$$

Considering this expansion as quantum mechanical Hamiltonian with $\hat{\boldsymbol{p}}=-i \hbar \nabla$, the second member of the right-hand side of the equation coincides with the nonrelativistic kinetic energy operator. However, this leads to a strange conclusion that the expectation value of the kinetic energy operator $\hat{\boldsymbol{p}}^{2} /(2 \mu)$, present in the "nonrelativistic" Schrödinger equation gives, according to the definition (56), an upper bound for the relativistic kinetic energy. At the same time, it is obvious that the expectation value of the relativistic kinetic energy has to be larger than the nonrelativistic at all values of the momentum. On the other hand, this means that the Schrödinger equation is not the nonrelativistic, because the present kinetic energy operator produces a kinetic energy of the particle larger than the relativistic one.

Moreover, the construction of the wave packet, moving, as necessary, with uniform velocity, given by the initial condition, is at all impossible. First, let us consider the nonrelativistic approximation. The analytical expression of the packet, completely analogous to the considered above packet with nonrelativistic momentum operator (45), is possible. Only the matter wave (33), initial condition (37) and spectral function (40) are modified, introducing instead of the operator $\boldsymbol{p}_{0}$ the relativistic momentum operator $\boldsymbol{p}$ and corresponding eigenvalues $p$ and $p_{i}$ : Finally, the packet is defined by the following expression:

$$
\frac{1}{\sigma(t) \sqrt{2 \pi}} \exp \left(-\frac{\left(z-\frac{p_{i}}{\mu} t\right)^{2}}{2 \sigma^{2}(t)}\right) \text {. }
$$

However, the velocity of the maximum of this probability distribution equals $p_{i} / \mu=v / \sqrt{1-v^{2} / c^{2}}$ and does not coincide with the particle velocity $v$.

Taking the relativistic expression of the corresponding Hamiltonian (58), after 
numerical calculation we obtained analogous result-the velocity of the maximum of the relativistic packet does not coincide with the velocity of the particle. At some combinations of parameters of the initial condition it is larger and at some other-smaller than necessary.

In the ultrarelativistic situation, when $p c>\mu c^{2}$ (for proton beams in CERN accelerator $\frac{p c}{\mu c^{2}} \approx 10^{8}$, for some particles of cosmic rays this relation can take even larger values) the converging expansion for kinetic energy equals

$$
T=p c-\mu c^{2}+\mu c^{2} \sum_{k=0}^{\infty}(-1)^{k} \frac{(2 k-1) ! !}{(2 k+2) ! !}\left(\frac{\mu c}{p}\right)^{2 k+1},
$$

thus the two first members of the expansion applied for packet construction produce matter waves and packets, moving at the speed of light. Therefore, again we have a strange result, not satisfying elementary conditions of special relativity theory.

The packet, satisfying mentioned conditions, for this momentum operator definition can be obtained only at extremely large $\sigma$, when the bell-shaped spectral function

$$
\exp \left(-\frac{\sigma^{2}\left(p-p_{i}\right)^{2}}{\hbar^{2}}\right)
$$

defines values of momentum $p$ distributed in close vicinity of initial $p_{i}$ and relativistic kinetic energy can be expressed with necessary precision as

$$
T(p)=T\left(p_{i}\right)+\left.\frac{\mathrm{d} T(p)}{\mathrm{d} p}\right|_{p=p_{i}}\left(p-p_{i}\right) .
$$

Due to definition (56) it follows

$$
\left.\frac{\mathrm{d} T(p)}{\mathrm{d} p}\right|_{p=p_{i}}=\frac{p_{i}}{\mu}\left(1+\left(\frac{p_{i}}{\mu c}\right)^{2}\right)^{-1 / 2}=\frac{p_{0 i}}{\mu}
$$

and the packet moves like the particle. However, the obtained results are only more or less successful approximations, not giving a precise solution of this simplest problem of quantum mechanics.

\section{Matter Waves and Packets, Satisfying Necessary Conditions}

Two the best known relativistic kinetic energy presentations in terms of nonrelativistic momentum $\boldsymbol{p}_{0}$ and in terms of relativistic momentum $\boldsymbol{p}$ were applied together with corresponding nonrelativistic approximations. However, both definitions of the quantum momentum operator, applied in these expressions, gives more or less acceptable approximations, but cannot present a precise solution for the problem of the free quantum particle. Thus, a definition of the new correspondence of this operator to some classical parameter with momentum dimension is necessary.

This problem is solved using relativistic kinetic energy (6), presented in terms 
of the nonrelativistic kinetic energy as

$$
T=\frac{p_{0}^{2}}{2 \mu} g(\beta),
$$

where

$$
g(\beta)=\sum_{k=0}^{\infty}\left(\begin{array}{c}
2 k+1 \\
k
\end{array}\right)\left(\frac{\beta}{4}\right)^{k}
$$

and $\beta=v^{2} / c^{2}$.

Defining

$$
\tilde{\boldsymbol{p}}=\boldsymbol{p}_{0} g(\beta)
$$

the relativistic kinetic energy can be present in form

$$
T=\frac{\tilde{p}^{2}}{2 \mu g(\beta)} .
$$

Now, for a precise description of the free particle, it is enough to define the quantum mechanical momentum operator $-i \hbar \nabla$ to correspond to the modified momentum of classical relativity theory $\tilde{\boldsymbol{p}}$.

This definition does not look strange and is completely acceptable in comparison with the traditional correspondence of this operator to

$$
\boldsymbol{p}=\boldsymbol{p}_{0} \gamma(\beta)
$$

with

$$
\gamma(\beta)=\sum_{k=0}^{\infty}\left(\begin{array}{c}
2 k \\
k
\end{array}\right)\left(\frac{\beta}{4}\right)^{k}
$$

Therefore, the difference of definitions present in Equation (65) and Equation (69) cannot be considered significant. Both momenta at nonrelativistic velocities are equal the nonrelativistic momentum, both are defined only at $0 \leq \beta<1$ and both approach infinity at velocity of particle approaching speed of light.

The new definition of momentum operator correspondence gives matter waves-solutions of the Schrödinger equation-whose packet produces the probability distribution of a free particle, moving in an inertial reference frame with given uniform velocity $0 \leq v<c$. Now the probability density distribution $|\psi(z, t)|^{2}$ in complete analogy with (45) is

$$
\frac{1}{\sigma(t) \sqrt{2 \pi}} \exp \left(-\frac{(z-v t)^{2}}{2 \sigma^{2}(t)}\right) \text {. }
$$

The only difference is that the spatial extension of the packet takes the form

$$
\sigma(t)=\sigma \sqrt{1+\left(\frac{\hbar t}{2 \mu g(\beta) \sigma^{2}}\right)^{2}}
$$

with multiplier $g(\beta)>1$, reducing the relativistic packet spreading.

Let us now evaluate this effect in some real situations. From definition 


$$
\gamma(\beta)=\frac{1}{\sqrt{1-\beta}}=\frac{E}{\mu c^{2}}
$$

it follows that

$$
\beta=1-\left(\frac{\mu c^{2}}{E}\right)^{2}
$$

and, after explicit summation of the expansion, present in Equation (65), $g(\beta)$ takes the simple form:

$$
g(\beta)=\frac{2}{\beta}\left(\frac{1}{\sqrt{1-\beta}}-1\right)
$$

The first problems let us investigate how much time $t_{0}$ is necessary for extension doubling, that is for relation

$$
\sigma\left(t_{0}\right)=2 \sigma
$$

which is satisfied by an electron, produced in the Stanford linear accelerator and a proton, produced in the CERN accelerator.

Both bunches are $\sigma \approx 1 \mathrm{~cm}$ in length (see Particle Data Group report [10] and earlier reports of this group). For electron $\left(E=50 \mathrm{GeV}, \mu c^{2}=511 \mathrm{keV}\right)$ one obtains

$$
\beta \approx 1-10^{-10}
$$

therefore

$$
g(\beta) \approx 2 \times 10^{5}
$$

and

$$
t_{0} \approx 5 \times 10^{5} \mathrm{~s}
$$

During this time the electron covers a distance

$$
l_{0} \approx 10^{14} \mathrm{~m} \text {. }
$$

For proton $\left(E=7 \mathrm{TeV}, \mu c^{2}=938 \mathrm{MeV}\right)$ the corresponding distance is even larger, equal approximately 3 light years $\left(1 \mathrm{ly}=0.946 \times 10^{16} \mathrm{~m}\right)$.

The obtained results create new possibilities to explain traveling of cosmic rays. The shortest super high energy event in space is a supernova explosion, lasting approximately one second. If this equals the spreading of the corresponding packet, the produced protons until $\sigma$ doubling cover distance, equal some $10^{20} \mathrm{ly}$ (for electrons this evaluation gives a slightly smaller value, equal $\left.10^{18} \mathrm{ly}\right)$. These distances are at least 100 million times larger than the dimension of the visual universe $\left(1.38 \times 10^{10} \mathrm{ly}\right)$, thus the spreading of the corresponding packet in the real universe is negligible. This effect can explain the unclear origin of extremely high energies, obtained registering these events.

\section{Conclusions}

From our consideration, it follows that the traditional definition of the quantum momentum operator, correspondence to the classical relativistic momentum 
cannot produce packets, moving in an inertial reference frame with uniform velocity less than the speed of light so that the maximal probability density point of the packet moves in the same way as a classical relativistic particle.

While solving this problem, a simple expression for the relativistic kinetic energy operator is defined, after postulating the original correspondence of the known quantum mechanical momentum operator $-i \hbar \nabla$ to a new factor with momentum dimension of classical relativity theory. This correspondence is necessary to present the relativistic kinetic energy operator in a form, satisfying the requirements for a matter particle wave packet. Schrödinger equation for a free particle with this kinetic energy operator gives a solution for the matter waves, whose packet moves in complete accordance with special relativity theory predictions for a free particle movement.

The developed theory significantly reduces spreading of packets, created after gigantic energy processes in universe, explaining extreme energies of cosmic rays, moving across the visible universe like compact bunches without remarkable spreading.

Moreover, the introduced relativistic kinetic energy operators can be applied in the internal Hamiltonian instead of nonrelativistic operators for every internal (Jacobian) variable according to the recipe, given in Sec. II. Both operators are differential of the same kind, the difference is only in the reduced mass of corresponding clusters, defined by given coordinate. The structure of this operator for relative movement of two particles or clusters is:

$$
T(\beta)=T(0) / g(\beta),
$$

where

$$
T(0)=-\frac{\hbar^{2}}{2 \mu} \Delta
$$

is nonrelativistic kinetic energy operator, $\mu$ is reduced mass of clusters, and $\Delta$ is Laplace operator, dependent on radius vector $\boldsymbol{r}$, connecting the centers of mass of clusters.

The nonrelativistic Schrödinger equation is

$$
(T(0)+V) \Psi=E \Psi .
$$

The equation with relativistic kinetic energy operator looks as

$$
(T(\beta)+V) \Psi=E \Psi
$$

or

$$
(T(0)+g(\beta) V) \Psi=g(\beta) E \Psi .
$$

Having in mind that $g(\beta) \geq 1$ one arrives at an interesting conclusion that a relativistic system has larger binding energy than the corresponding nonrelativistic system, mainly due to a larger effective reduced mass of interacting particles, equal $\mu g(\beta)$.

For a two-particle quantum system, the parameter $\beta$ can be present in form 


$$
\beta=\frac{2\langle T(0)\rangle}{\mu c^{2}}
$$

where $\langle T(0)\rangle$ marks the expectation value of nonrelativistic kinetic energy operator.

Let us evaluate this effect for the Hydrogen atom. From virial theorem, it follows that

$$
\langle T(0)\rangle=-E \approx 13.6 \mathrm{eV}
$$

therefore

$$
\beta \approx \frac{27.2 \mathrm{eV}}{511 \mathrm{keV}} \approx 5 \times 10^{-5}
$$

and

$$
g(\beta) \approx 1.00004 .
$$

Thus, for Hydrogen atom, the relativistic correction of the binding energy is negligible.

For deuteron the kinetic energy expectation value, obtained after Schrödinger equation with Argonne $\mathrm{v}_{18}$ realistic potential solution, equals $19.81 \mathrm{MeV}$ [11]. This value, according to (85), gives $\beta \approx 0.084$, therefore $g(\beta) \approx 1.068$. From Equation (84) it follows that the corrected value $g(\beta) E$ of deuteron binding energy is $2.3759 \mathrm{MeV}$. Argonne and other modern realistic potentials are fitted to experimental deuteron binding energy, equal $2.2246 \mathrm{MeV}$. Therefore, taken into account relativistic nucleon mass correction requires more attractive potential in comparison to known potentials in a two-nucleon channel ${ }^{3} S_{1}-{ }^{3} D_{1}$. This gives us a chance to solve the old problem of realistic potentials, carefully fitted with the two-nucleon data, giving smaller than experimental binding energies of more complex nuclei.

Obviously, the relativistic effects, taken into account in the defined way, can be even more significant when particles are trapped in a restricted space, like the quarks in a nucleon or protons in a protostar. Our definition of the relativistic kinetic energy gives a possibility for some kind of confinement to occur in these systems, preventing disintegration of the system when the attractive force increases with the growing kinetic energy of the relative movement of particles or clusters. Moreover, if inertial and gravitational masses are equal, gravity, caused by the obtained mass dependence on velocity, can become significant for relativistic particles.

\section{Conflicts of Interest}

The author declares no conflicts of interest regarding the publication of this paper.

\section{References}

[1] Kamuntavičius, G.P. (1989) Reduced Hamiltonian Method for Many-Particle Quantum Systems Bound States Theory. Soviet Journal Particles and Nuclei, 20, 
109-120 [Fiz. Elemen. Chastits At. Yadra, 20, 261-292].

[2] Navratil, P., Kamuntavičius, G.P. and Barrett, B.R. (2000) Few-Nucleon Systems in a Translationally Invariant Harmonic Oscillator Basis. Physical Review C, 61, Article ID: 044001. https://doi.org/10.1103/PhysRevC.61.044001

[3] Kamuntavičius, G.P. (2014) Galilei Invariant Technique for Quantum System Description. Journal of Mathematical Physics, 55, Article ID: 042103. https://doi.org/10.1063/1.4870617

[4] Fodor, Z. and Hoelbling, C. (2012) Light Hadron Masses from Lattice QCD. Reviews of Modern Physics, 84, 449-495. https://doi.org/10.1103/RevModPhys.84.449

[5] Messiah, A. (1965) Quantum Mechanics. Vol. 1, North-Holland, Amsterdam.

[6] Goldberger, M.L. and Watson, K.M. (1964) Collision Theory. John Wiley \& Sons, NY.

[7] Brandt, S. and Dahmen, H.D. (1995) The Picture Book of Quantum Mechanics. 2nd Edition, Springer-Verlag, NY. https://doi.org/10.1007/978-1-4684-0233-9

[8] Kamuntavičius, G.P. and Kamuntavičius, G. (2017) Kinematic Relativity of Quantum Mechanics: Free Particle with Different Boundary Conditions. Journal of Applied Mathematics and Physics, 5, 853-861. https://doi.org/10.4236/jamp.2017.54075

[9] Kamuntavičius, G.P. and Kamuntavičius, G. (2017) Relativistic Dynamics of a Quantum System. Journal of Applied Mathematics and Physics, 5, 1478-1492. https://doi.org/10.4236/jamp.2017.57121

[10] Tanabashi, M., et al. (Particle Data Group) (2018) Review of Particle Physics. Physical Review D, 40, Article ID: 030001. https://doi.org/10.1103/PhysRevD.98.030001

[11] Forest, J.L., Pandharipande, V.R. and Arriaga, A. (1999) Quantum Monte Carlo Studies of Relativistic Effects in Light Nuclei. Physical Review C, 60, Article ID: 014002. https://doi.org/10.1103/PhysRevC.60.014002 